\title{
Comparison of serum markers for allergic bronchopulmonary aspergillosis in cystic
}

\section{fibrosis}

\author{
P. Latzin**,ף, D. Hart| ${ }^{\#, \uparrow, ~ N . ~ R e g a m e y *, ~ U . ~ F r e y *, ~ M . H . ~ S c h o e n i * ~ a n d ~ C . ~ C a s a u l t a * ~}$
}

ABSTRACT: The diagnosis of allergic bronchopulmonary aspergillosis (ABPA) in cystic fibrosis (CF) is a challenge. Thymus- and activation-regulated chemokine (TARC) has recently been reported to play a role in ABPA. The aim of this study was to compare the diagnostic value of TARC with that of known serological markers for diagnosis of ABPA in CF patients.

The present study longitudinally followed 48 CF patients, of whom 12 had a diagnosis of ABPA according to Nelson's criteria, for 1-8 yrs with repeated measurements of serum total immunoglobulin (Ig)E, specific Aspergillus fumigatus IgE and IgG, specific IgE against recombinant $A$. fumigatus allergens (rAsp f) 1, 3, 4 and 6, and TARC.

Median (interquartile range) TARC levels were 589 (465-673) $\mathrm{pg} \cdot \mathrm{mL}^{-1}$ in ABPA patients and 232 (189-289) $\mathrm{pg} \cdot \mathrm{mL}^{-1}$ in non-ABPA patients. Receiver operating characteristic curves revealed that TARC was superior to the other markers for diagnosis of ABPA. Diagnostic accuracy was greater for TARC (93\%) than for total IgE (74\%), or rAsp f $4(75 \%)$ or $f 6(79 \%)$.

The present study indicates that thymus- and activation-regulated chemokine may be useful in the diagnosis of allergic bronchopulmonary aspergillosis in cystic fibrosis patients. However, larger studies are needed before thymus- and activation-regulated chemokine can routinely be used in diagnostic algorithms.

KEYWORDS: Allergic bronchopulmonary aspergillosis, cystic fibrosis, diagnostic value, immunoglobulin E, serum marker, thymus- and activation-regulated chemokine

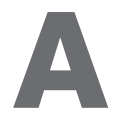
llergic bronchopulmonary aspergillosis (ABPA) is a pulmonary hypersensitivity disease mediated by an allergic response to Aspergillus fumigatus [1]. ABPA occurs in $~ 10 \%$ of cystic fibrosis (CF) patients and may lead to acute worsening of respiratory status and ongoing decline in lung function [2], ultimately progressing to a chronic state and lung fibrosis without adequate treatment [3]. Despite the existence of the gold-standard Nelson criteria [4], diagnosis of ABPA in CF patients remains difficult [5]. The wide variation in diagnostic practices between clinics [6], different estimates of prevalence and a delay in recognition lead to undertreatment [7].

The main reason for the difficulties in diagnosis of ABPA and ABPA exacerbations in CF patients is the overlap of diagnostic criteria for ABPA with common manifestations of CF. Pulmonary infiltrates, obstructive lung disease and bronchiectasis occur regularly in CF patients, due to the underlying disease with bacterial colonisation, and thus are not specific to ABPA [8]. Furthermore, lung colonisation with $A$. fumigatus occurs in $20-25 \%$ of CF patients [9-11]. Therefore, as stated in the most recent consensus document on diagnosis and therapy of ABPA in CF patients, serological findings should contribute strongly to the confirmation or exclusion of clinically suspected ABPA [5].

Animal studies suggest a pathophysiological role of the chemokine thymus- and activation-regulated chemokine (TARC) in ABPA by linking an antifungal immune response with the promotion of T-helper cell (Th) type 2-mediated hypersensitivity to A. fumigatus [12]. It has recently been shown, in a cross-sectional study, that serum levels of TARC are elevated in CF patients with ABPA [13]. However, longitudinal clinical data on the usefulness of this marker are lacking and no further study has been performed validating TARC in comparison to other putative ABPA serum markers. The present study aimed to answer the following questions. 1) Can the results of the previously published cross-sectional study [13] be confirmed in another CF population?

\section{AFFILIATIONS}

*Division of Respiratory Medicine, Children's University Hospital of Bern, Bern, Switzerland,

\#Children's Hospital of the Ludwig Maximilian University of Munich, Munich, Germany.

'Both authors contributed equally to this article.

\section{CORRESPONDENCE}

P. Latzin

Division of Respiratory Medicine Dept of Paediatrics

University Children's Hospital of Bern Inselspital

3010 Bern

Switzerland

Fax: 41316324807

E-mail: philipp.latzin@insel.ch

Received:

June 262007

Accepted after revision:

September 112007

\section{SUPPORT STATEMENT}

This study was supported by a Swiss National Foundation (Berne,

Switzerland) grant 3200-B0-112099

to U. Frey, a grant from the German Society for Paediatric Pneumology

(Hanover, Germany) to D. Hartl and a grant from the Münchener Medizinische Wochenschrift (Munich, Germany) to P. Latzin.

STATEMENT OF INTEREST

None declared. 
2) What is the diagnostic value (sensitivity and specificity) of TARC compared to other serological markers of ABPA? 3) Is TARC useful for the early detection of ABPA development?

\section{METHODS}

\section{Study design}

From 1998 onwards, a group of 48 patients ( 23 females and 25 males; median (interquartile range (IQR)) age 9 (7-14) yrs) with CF were systematically followed longitudinally [14]. All patients underwent careful clinical assessment, lung function testing and microbiological diagnosis at all visits during the study period. Skin testing against $A$. fumigatus was routinely performed using Bencard skin test antigens (SmithKline Beecham, Münchenbuchsee, Switzerland). Chest radiography was performed on study entry and thereafter at least annually.

In addition, serum samples were collected at the study visits, resulting in an average of six serum samples per patient for analysis (table 1). The Ethics Committee of Berne (Berne, Switzerland) approved the study and written consent was obtained on enrolment.

\section{Clinical diagnosis of ABPA}

According to Nelson's criteria, patients were diagnosed as having clinical ABPA when at least six out of the seven following criteria were fulfilled: wheezing, positive A. fumigatus sputum culture, presence of defined infiltrates on chest radiography, positive acute reaction to $A$. fumigatus on

\begin{tabular}{|c|c|c|c|}
\hline \multirow[t]{2}{*}{ TABLE 1} & \multicolumn{3}{|c|}{$\begin{array}{l}\text { Patient data and results of serum measurements } \\
\text { in cystic fibrosis patients with and without allergic } \\
\text { bronchopulmonary aspergillosis (ABPA) }\end{array}$} \\
\hline & & ABPA & Non-ABPA \\
\hline \multicolumn{2}{|l|}{ Patients } & 12 & 36 \\
\hline \multicolumn{2}{|c|}{ Age at study entry yrs } & $10(8-12)$ & $9(7-14)$ \\
\hline \multicolumn{2}{|c|}{ Males/females } & $7 / 5$ & $19 / 17$ \\
\hline \multicolumn{2}{|c|}{ Study duration months } & $40(35-80)$ & $40(27-55)$ \\
\hline \multicolumn{2}{|c|}{ Serum samples } & $7(4-12) ; 87^{+}$ & $5(1-8) ; 178^{+}$ \\
\hline \multicolumn{2}{|c|}{ FEV 1 on study entry $\%$ pred } & $73(77-81)$ & 89 (72-99) \\
\hline \multicolumn{2}{|c|}{ A. fumigatus in sputum ${ }^{\#}$} & 12 & 20 \\
\hline \multicolumn{2}{|c|}{$P$. aeruginosa in sputum ${ }^{\#}$} & 12 & 34 \\
\hline \multicolumn{4}{|c|}{ Serum measurements } \\
\hline \multicolumn{2}{|c|}{ Total IgE IU $\mathrm{mL}^{-1}$} & 965 (324-1961) & $59(21-433)$ \\
\hline \multicolumn{2}{|c|}{ Specific IgG to $A$. fumigatus $\mathrm{kU} \cdot \mathrm{L}^{-1}$} & $145(66-244)$ & $51(17-105)$ \\
\hline \multicolumn{2}{|c|}{ Specific IgE to A. fumigatus RAST class" } & $4(3-5)$ & $1(0-3)$ \\
\hline \multicolumn{2}{|c|}{$\mathrm{rAsp} f 1 \mathrm{EU} \cdot \mathrm{mL}^{-1}$} & $128(60-364)$ & $21(6-59)$ \\
\hline \multicolumn{2}{|c|}{$\mathrm{rAsp} f 3 \mathrm{EU} \cdot \mathrm{mL}^{-1}$} & $253(99-739)$ & $37(14-94)$ \\
\hline \multicolumn{2}{|c|}{$\mathrm{rAsp} f \mathrm{EU} \cdot \mathrm{mL}^{-1}$} & $23(11-53)$ & $4(1-12)$ \\
\hline \multicolumn{2}{|c|}{$\mathrm{rAsp} f\left(6 \mathrm{EU} \cdot \mathrm{mL}^{-1}\right.$} & $25(8-67)$ & $3(2-8)$ \\
\hline \multicolumn{2}{|c|}{$\mathrm{TARC} \mathrm{pg} \cdot \mathrm{mL}^{-1}$} & $589(465-673)$ & $232(189-289)$ \\
\hline
\end{tabular}

Data are presented as $n$ or median (interquartile range) unless otherwise stated. FEV1: forced expiratory volume in one second; \% pred: \% predicted; A. fumigatus: Aspergillus fumigatus; $P$. aeruginosa: Pseudomonas aeruginosa; Ig: immunoglobulin; RAST: radioallergosorbent test; rAsp f: recombinant $A$. fumigatus allergen; EU: ELISA unit; TARC: thymus- and activation-regulated chemokine. ${ }^{*}$ : at least one positive sample during the study period; ": 0-6; ${ }^{+}$: median (range) per patient; total number of samples. skin-prick testing, elevated total immunoglobulin (Ig)E levels (cut-off $500 \mathrm{IU} \cdot \mathrm{mL}^{-1}$ ), increased levels of specific serum $\mathrm{IgE}$ (cut-off $17.5 \mathrm{IU} \cdot \mathrm{mL}^{-1}$ ) and $\operatorname{IgG}$ (cut-off $20 \mathrm{kU} \cdot \mathrm{L}^{-1}$ ) directed against $A$. fumigatus [4].

\section{Serum markers}

In the collected serum samples, the following parameters were measured: total IgE, specific IgE (radioallergosorbent test) to $A$. fumigatus, specific IgG to $A$. fumigatus extract (ELISA) and specific IgE against the recombinant $A$. fumigatus allergens (rAsp f) 1, 3, 4 and 6 [14, 15] and TARC.

TARC levels were analysed in triplicate by sandwich ELISA (R\&D Systems, Minneapolis, MN, USA) according to the manufacturer's instructions, and concentrations calculated from standard curves with detection limits of 7-3,000 $\mathrm{pg} \cdot \mathrm{mL}^{-1}$. Intraassay variability was determined by evaluating five serum samples 10 times within the same assay run and showed a coefficient of variation of $6-9 \%$. Interassay variability was determined by measuring five serum samples in five consecutive assay runs and showed a coefficient of variation of $8-17 \%$.

\section{Statistical analysis}

Data are presented as median (IQR) unless otherwise indicated. The diagnostic value of the serological markers and receiver operator characteristic (ROC) curves were calculated. Cut-off levels were set at the level that resulted in the optimal diagnostic accuracy, defined as correctly positively classified plus correctly negatively classified as a percentage of the total.

\section{Nested matched case-control analysis}

In order to assess whether TARC level elevation was specific to ABPA or an epiphenomenon of the hypersensitivity against A. fumigatus in ABPA patients, a nested matched case-control analysis was performed and TARC levels compared between cases (ABPA patients) and controls (non-ABPA patients), matched for total IgE and rAsp f 6 levels, respectively.

\section{RESULTS}

Of the 48 CF patients, 12 were diagnosed with clinical ABPA based on Nelson's criteria. Nine patients were diagnosed with ABPA before study entry and three developed their first episode of clinical ABPA during the study period; all were assigned to the ABPA group. The other $36 \mathrm{CF}$ patients did not fulfil six out of seven of Nelson's criteria for diagnosis at any time before or during the study period and were assigned to the non-ABPA group. Details of the patients' characteristics are given in table 1 .

\section{TARC compared to other serological markers for the diagnosis of ABPA}

Median (IQR) TARC levels were $589(465-673) \mathrm{pg} \cdot \mathrm{mL}^{-1}$ in ABPA patients compared to 232 (189-289) $\mathrm{pg} \cdot \mathrm{mL}^{-1}$ in nonABPA patients (table 1). In the 16 non-ABPA patients with neither sensitisation to $A$. fumigatus nor elevation of total $\mathrm{IgE}$ levels, TARC levels were $207(178-282) \mathrm{pg} \cdot \mathrm{mL}^{-1}$.

When the serological results of all available time points in the study period were included $(n=265)$, the sensitivity, specificity and diagnostic accuracy for diagnosis of ABPA were as follows: 92,95 and $93 \%$ for TARC; 65,81 and $74 \%$ for total 
TABLE 2 Value of the various markers for allergic bronchopulmonary aspergillosis (ABPA) diagnosis using all serum samples

\begin{tabular}{|c|c|c|c|c|c|c|c|}
\hline Cut-off level EU $\cdot \mathrm{mL}^{-1}$ & $386^{\# \#}$ & $514^{\circ \pi}$ & 75 & 140 & 10 & 16 & $140^{++}$ \\
\hline Specificity \% & 94.7 & 81.0 & 83.3 & 85.9 & 71.2 & 86.5 & 83.7 \\
\hline Diagnostic accuracy $^{+} \%$ & 93.4 & 74.3 & 78.1 & 79.0 & 74.8 & 79.0 & 73.2 \\
\hline Positive likelihood ratio ${ }^{\S}$ & 17.3 & 3.4 & 4.1 & 4.7 & 2.8 & 4.8 & 3.3 \\
\hline
\end{tabular}

TARC: thymus- and activation-regulated chemokine; Ig: immunoglobulin; rAsp f: recombinant Aspergillus fumigatus allergen; EU: ELISA unit. \#: the probability that a patient with ABPA shows elevated serum levels relative to the cut-off level of the relevant marker; ": the probability that a patient without ABPA shows serum levels below the cut-off level of the relevant marker; ${ }^{+}$: the number of correctly positively categorised plus correctly negatively categorised patients as a percentage of the total; ${ }^{\text {s: }}$ the true-positive rate divided by the false-positive rate (a higher ratio indicates a better test) ${ }^{f}$ : the false-negative rate divided by the true-negative rate (a lower ratio indicates a better test); ${ }^{\# \#}: \mathrm{pg} \cdot \mathrm{mL}^{-1} ;{ }^{\bullet}: \mathrm{IU} \cdot \mathrm{mL}^{-1} ;{ }^{++}: \mathrm{KU} \cdot \mathrm{L}^{-1}$

IgE; 68,83 and $78 \%$ for rAsp f $1 ; 66,86$ and $79 \%$ for rAsp f $3 ; 82$, 71 and $75 \%$ for rAsp f $4 ; 65,87$ and $79 \%$ for rAsp f 6 ; and 54,84 and $73 \%$ for IgG (table 2 ).

These results were confirmed using ROC curve analysis, with TARC levels resulting in the largest area under the curve, as illustrated in figure 1 for TARC, total IgE, rAsp f 4 and rAsp f 6 .

In a second approach, only one serum sample per patient was used for analysis, namely that from the time point with the highest total IgE level. Again, TARC levels resulted in the greatest diagnostic accuracy, sensitivity and specificity, as well as the largest area under the curve (table 3).

\section{Nested matched case-control analysis}

In the nested matched case-control design, TARC levels discriminated well between 10 patients with clinical ABPA (cases) and 10 without clinical ABPA (controls), matched for

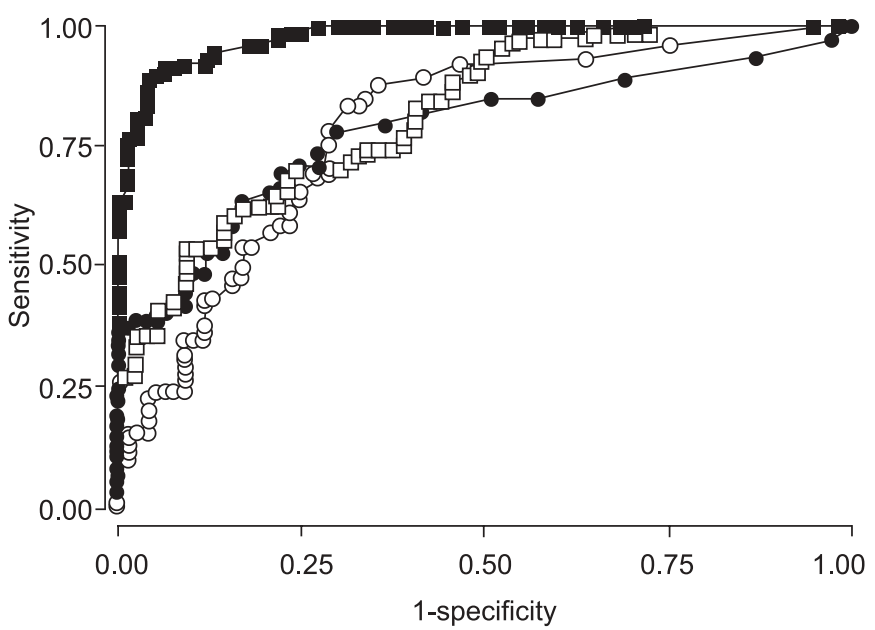

FIGURE 1. Receiver operating characteristic curves for thymus- and activation-regulated chemokine (TARC; $\boldsymbol{\square})$, total immunoglobulin (Ig)E ( $\square$ ), recombinant Aspergillus fumigatus allergens $(\mathrm{rAsp}$ f) $4(\bigcirc)$ and $6(\bullet)$ for diagnosis of allergic bronchopulmonary aspergillosis (ABPA). All available serum samples of the cohort $(n=265)$ were used. The resulting areas under the curve were 0.98 for TARC, 0.84 for total lgE, 0.79 for rAsp $f 4$ and 0.80 for rAsp $f 6$. total IgE level (median total IgE level of 1,375 IU $\cdot \mathrm{mL}^{-1}$ for the ABPA patients and $1,152 \mathrm{IU} \cdot \mathrm{mL}^{-1}$ for the non-ABPA patients). The median TARC level was $673 \mathrm{pg} \cdot \mathrm{mL}^{-1}$ for the ABPA patients compared to $237 \mathrm{pg} \cdot \mathrm{mL}^{-1}$ for non-ABPA patients (fig. 2). Two ABPA patients could not be included in the casecontrol analysis due to very high serum IgE levels (3,710 and $5,263 \mathrm{IU} \cdot \mathrm{mL}^{-1}$ ) without matching controls. These patients showed elevated TARC levels of 432 and $721 \mathrm{pg} \cdot \mathrm{mL}^{-1}$, respectively.

TARC levels also discriminated well between 11 ABPA patients (median TARC level $566 \mathrm{pg} \cdot \mathrm{mL}^{-1}$ ) and 11 patients without ABPA (median TARC level $234 \mathrm{pg} \cdot \mathrm{mL}^{-1}$ ) matched for rAsp f 6 levels (median rAsp f 6 level of 13 ELISA units $(\mathrm{EU}) \cdot \mathrm{mL}^{-1}$ for the ABPA patients and $19 \mathrm{EU} \cdot \mathrm{mL}^{-1}$ for the nonABPA patients; fig. 3). One ABPA patient could not be included in the case-control analysis due to very high rAsp $\mathrm{f}$ 6 levels $\left(165 \mathrm{EU} \cdot \mathrm{mL}^{-1}\right)$ without matching control. This patient showed elevated TARC levels of $630 \mathrm{pg} \cdot \mathrm{mL}^{-1}$.

\section{Early elevation of TARC levels in the course of ABPA}

In the subgroup of the three CF patients who developed their first episode of clinical ABPA during the study period, TARC levels were elevated prior to development of the full clinical picture of ABPA and before total IgE level elevation (fig. 4).

\section{DISCUSSION}

The present study confirms elevated serum TARC levels in ABPA patients in a CF population not previously investigated and shows that TARC is a highly sensitive and specific marker for discrimination of ABPA patients in comparison with other serum markers. The present results further suggest that TARC levels may be elevated early in the course of ABPA in $\mathrm{CF}$ patients.

In a pilot study investigating chemokines and cytokines in ABPA, serum TARC levels were elevated in CF and asthma patients with ABPA compared to several $\mathrm{CF}$ and non-CF control groups [13]. However, in this study, chemokines were measured at different time-points in seven patients $\leqslant 4$ months before and after an ABPA exacerbation only. It is now possible to confirm elevated TARC levels in ABPA patients in a different $\mathrm{CF}$ population followed over a much longer period. It 
TABLE 3 Value of the various markers for allergic bronchopulmonary aspergillosis (ABPA) diagnosis using peak total immunoglobulin (Ig)E serum samples

\begin{tabular}{|c|c|c|c|c|c|c|c|}
\hline & TARC & $\lg E$ & rAsp $f 1$ & rAsp $\mathrm{f} 3$ & rAsp f 4 & rAsp f 6 & $\lg G$ \\
\hline Cut-off level EU $\cdot \mathrm{mL}^{-1}$ & $487^{\# \#}$ & $1624^{\circ}$ & 274 & 739 & 51 & 65 & $87^{++}$ \\
\hline Sensitivity ${ }^{\#} \%$ & 100 & 83.3 & 63.6 & 54.6 & 54.5 & 72.7 & 90.9 \\
\hline Diagnostic accuracy ${ }^{+} \%$ & 97.9 & 91.7 & 90.5 & 88.1 & 80.9 & 92.9 & 87.5 \\
\hline Positive likelihood ratio ${ }^{\S}$ & 36.0 & 15.0 & 19.7 & 16.9 & 5.6 & 22.5 & 6.6 \\
\hline
\end{tabular}

TARC: thymus- and activation-regulated chemokine; rAsp f: recombinant Aspergillus fumigatus allergen; EU: ELISA unit; ROC: receiver operating characteristic. ${ }^{\# \text { : the }}$ probability that a patient with ABPA shows elevated serum levels relative to the cut-off level of the relevant marker; ": the probability that a patient without ABPA shows serum levels below the cut-off level of the relevant marker; ${ }^{+}$: the number of correctly positively categorised plus correctly negatively categorised patients as a percentage of the total; ${ }^{\S}$ : the true-positive rate divided by the false-positive rate (a higher ratio indicates a better test); ${ }^{f}$ the false-negative rate divided by the true-negative rate (a lower ratio indicates a better test); ${ }^{\# \#}: \mathrm{pg} \cdot \mathrm{mL}^{-1} ;{ }^{\top}: \mathrm{IU} \cdot \mathrm{mL}^{-1} ;{ }^{++}: \mathrm{kU} \cdot \mathrm{L}^{-1}$.

is well known that patients from different $\mathrm{CF}$ populations and centres show great variability in their microbiological colonisation [9], atopic status [10] and genetic background [16]. Studies examining the role of genetic modifiers have also found diverse results among different CF populations [16]. Thus, the confirmation of elevated TARC levels over a longer period of time in ABPA patients in a second CF cohort is very important regarding the possible diagnostic use of TARC in a clinical setting.

The development of recombinant antibodies directed against various A. fumigatus allergens has facilitated ABPA diagnosis, and commercially available kits are now used for the assessment of sensitisation to A. fumigatus [17, 18]. Several approaches have been taken in the validation of various serological markers in order to simplify the diagnosis of ABPA $[14,15,17,19-23]$, mainly assessing the diagnostic value of recombinant Aspergillus antibodies but also looking at other serum markers, such as surfactant protein D [24]. Various groups have previously shown that the combined use of increased total $\mathrm{IgE}$ and $\mathrm{rAsp} \mathrm{f} 4$ and $\mathrm{f} 6$ permit reasonable discrimination between patients with ABPA and those with Aspergillus sensitisation without clinical ABPA [14, 21]. KURUP et al. [22] used both an ELISA and an ImmunoCAP® (Phadia $\mathrm{AB}$, Uppsala, Sweden) to determine rAsp f 1, 2, 3, 4 and 6 levels, and concluded that no single recombinant allergen is capable of differentiating between ABPA and non-ABPA patients. In the present study, TARC was compared to other

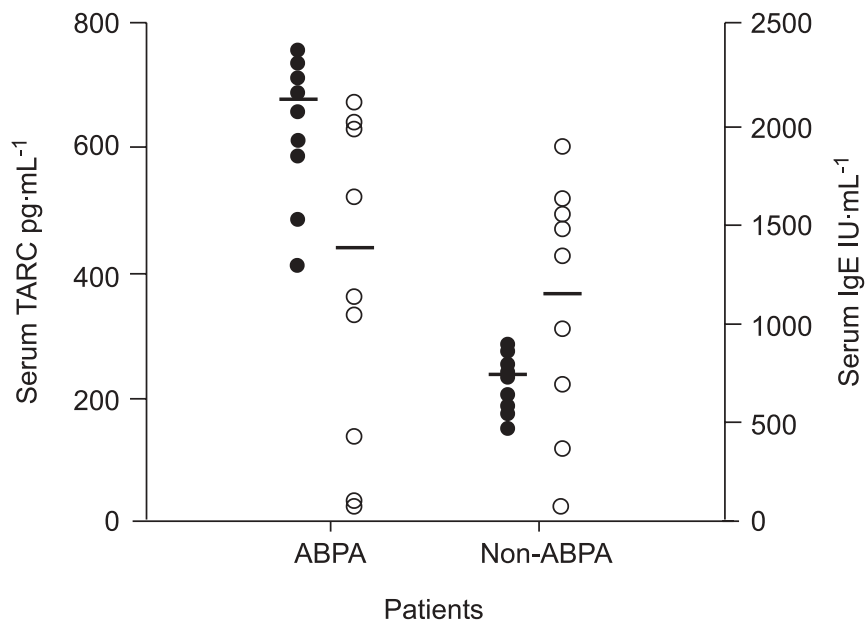

FIGURE 2. Nested case-control analysis: comparison of serum thymus- and activation-regulated chemokine (TARC; $\bullet$ ) levels in 10 allergic bronchopulmonary aspergillosis (ABPA; cases) and 10 non-ABPA cystic fibrosis patients (controls) matched for total serum immunoglobulin (Ig)E levels $(O)$. Two ABPA patients were excluded due to very high serum IgE levels $\left(3,710\right.$ and $\left.5,263 \mathrm{IU} \cdot \mathrm{mL}^{-1}\right)$ without matching control. Both showed elevated serum TARC levels (721 and $\left.432 \mathrm{pg} \cdot \mathrm{mL}^{-1}\right)$. Horizontal bars indicate medians

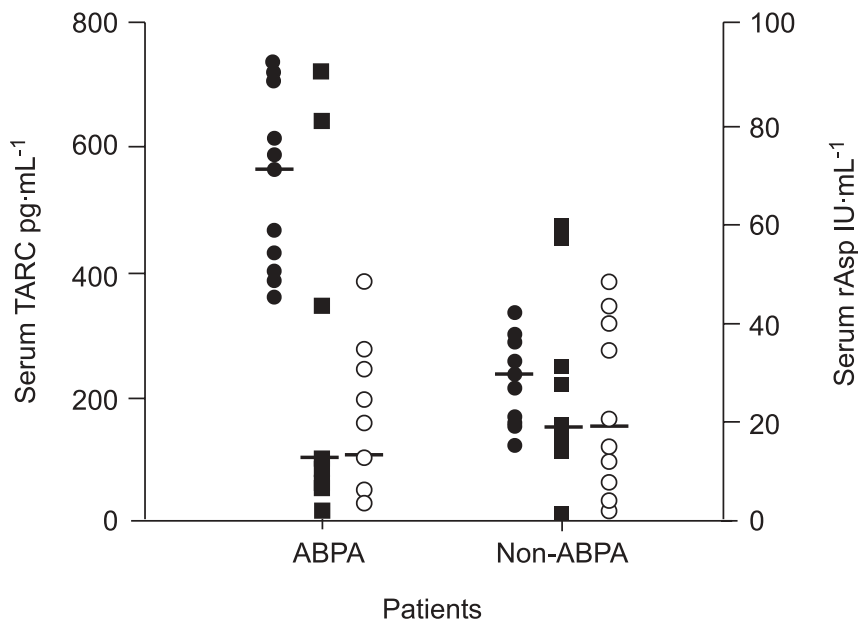

FIGURE 3. Nested case-control analysis: comparison of serum thymus- and activation-regulated chemokine (TARC; - ) levels in 11 allergic bronchopulmonary aspergillosis (ABPA; cases) and 11 non-ABPA cystic fibrosis patients (controls) matched for recombinant Aspergillus fumigatus allergen ( $r A s p$ f) 6 levels $(O)$. One ABPA patient was excluded due to very high rAsp $f 6$ levels (165 ELISA units $\cdot \mathrm{mL}^{-1}$ ) without matching control. This patient showed elevated serum TARC levels $\left(630 \mathrm{pg} \cdot \mathrm{mL}^{-1}\right)$. Horizontal bars indicate medians. $\mathbf{a}$ : rAsp f 4 

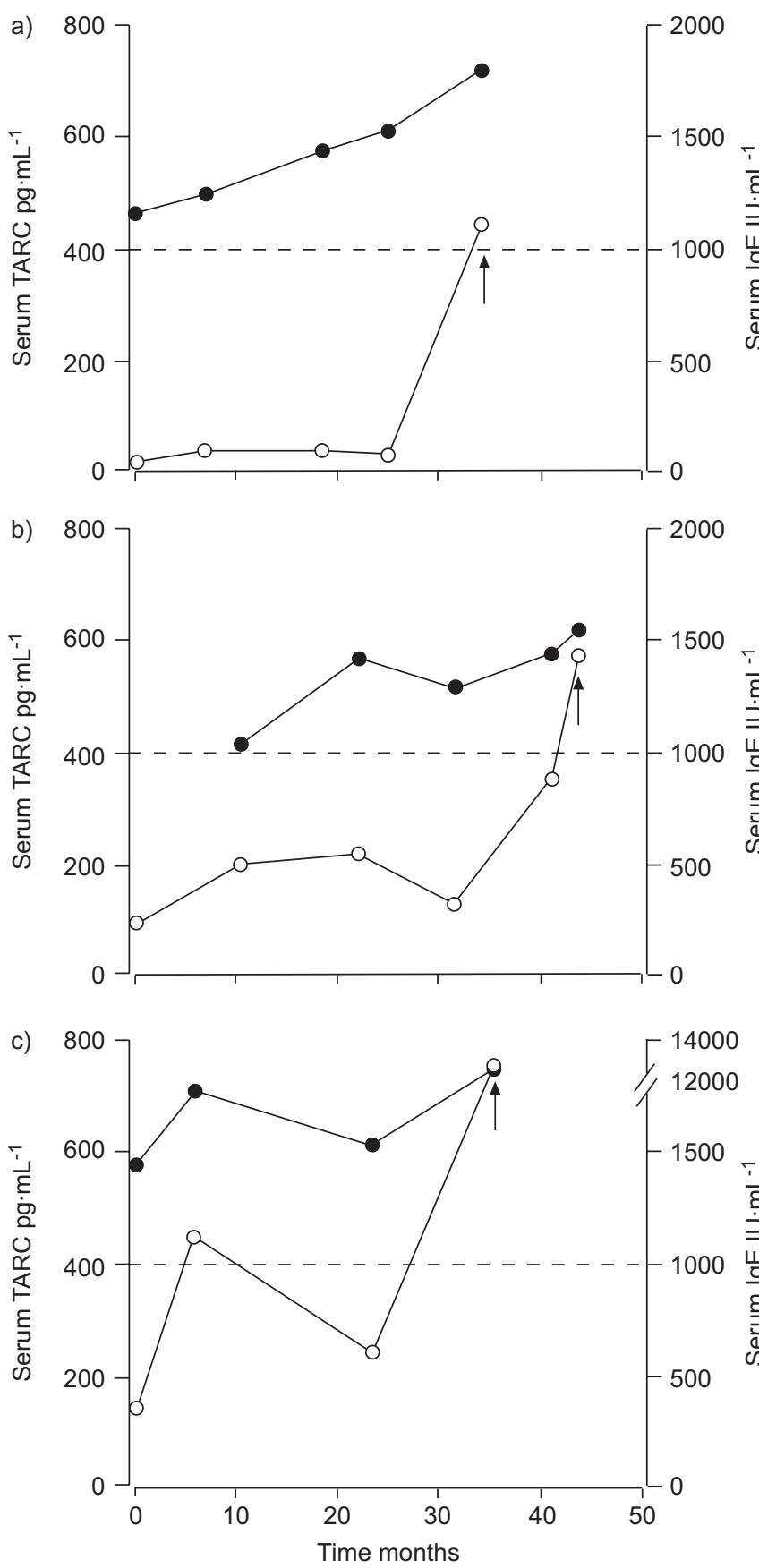

FIGURE 4. Longitudinal course of thymus- and activation-regulated chemokine (TARC; - levels compared to total immunoglobulin (Ig)E levels $(O)$ before the development of allergic bronchopulmonary aspergillosis (ABPA) in three individual patients $(a, b$ and $c)$ during the study period. Vertical arrow indicates clinical ABPA diagnosis. - - -: TARC cut-off level of $400 \mathrm{pg} \cdot \mathrm{mL}^{-1}$.

serological markers of ABPA using ROC curve analysis with two approaches. First, all serum samples from the whole study period were used for analysis. This reflects real clinical practice with longitudinal follow-up of CF patients. In a second approach, the diagnostic value of the various serological markers was evaluated using only the serum sample at the peak IgE level of each patient. The present findings of TARC as a single marker being clearly superior to other serological markers using both approaches highlight the potential of this new marker in clinical practice in contrast to complicated combination analysis of several serological markers [14, 22].

In the present cohort, the study design meant that the proportion of $\mathrm{CF}$ patients with ABPA was higher than in the typical CF population [11]. Therefore, the positive and negative predictive values of the various serological markers were not calculated, since such calculations take into account the prevalence of a disease in the study cohort. The high prevalence, however, does not weaken the strength of the present findings; on the contrary, despite this high prevalence, measuring TARC levels resulted in a very low negative likelihood ratio, indicating minimal false-negative classification compared to the other serum markers.

In the present study, the best cut-off value of TARC for discrimination of ABPA patients was $\sim 400 \mathrm{pg} \cdot \mathrm{mL}^{-1}$, depending on whether all serum samples or only the serum samples at the IgE peak were taken into account. Further studies are needed to determine optimal cut-off levels of TARC for the diagnosis of ABPA in CF, especially since such cut-off values may also be influenced by methodological issues and differ between patient populations, as is the case for IgE levels [5].

One patient without ABPA showed elevated TARC levels. To date, this patient has not fulfilled the clinical criteria of NELSON et al. [4] for diagnosis of ABPA, as detailed in the Clinical diagnosis of the ABPA section, but is being followed-up carefully. Thus it can only be speculated whether this patient will develop ABPA or whether TARC levels are elevated for other unknown reasons, such as the recently described entity of Aspergillus bronchitis in CF patients [25].

Although numbers were small, the present longitudinal study design permitted assessment of the value of TARC as an early marker of ABPA development in the subgroup of patients who experienced their first episode of clinical ABPA during the study period. TARC levels were elevated for up to 30 months before the clinical picture of ABPA and much earlier than total IgE levels. These results require confirmation in a larger number of patients but the early elevation of TARC levels makes this new marker even more interesting for potential clinical use, since the chances of misdiagnosis and long-term consequences decrease with early awareness of possible ABPA [1].

As reviewed recently by HARTL et al. [26], A. fumigatus conidia are recognised via Toll-like receptors on dendritic cells [27], which represent a major source of TARC [28]. TARC recruits Th2 cells via the chemokine receptor, CC chemokine receptor 4 , to the pulmonary site of inflammation [29]. Th2 cells produce interleukin-4, which induces IgE production by B-cells [30]. The secreted IgE, in turn, binds to mast cells, which release mediators ultimately leading to bronchoconstriction. Thus both the presence of $A$. fumigatus and the development of a Th2 response seem to be the underlying reasons for the strong TARC response in ABPA patients. As has previously been speculated for acute eosinophilic pneumonia [31] and allergic asthma [32], a pathophysiological role of TARC also seems very likely in the disease process of ABPA. However, the possibility cannot be excluded that TARC is only an epiphenomenon, indicating individual patients prone to 
developing ABPA, which should thus more correctly be termed a risk factor.

The IgE-independent mechanism of TARC activation might also explain the early increase in TARC compared to IgE levels, and the elevation of TARC levels independently of IgE levels in the nested matched case-control analysis.

Taken together, it has been shown that thymus- and activation-regulated chemokine levels are superior to those of other serum markers for the discrimination of cystic fibrosis patients with and without allergic bronchopulmonary aspergillosis followed longitudinally under clinical conditions. These findings might impact directly upon clinical practice, especially since thymus- and activation-regulated chemokine is a single marker and seems to be elevated early in the course of allergic bronchopulmonary aspergillosis. Before thymusand activation-regulated chemokine can be included in diagnostic algorithms for allergic bronchopulmonary aspergillosis in cystic fibrosis patients, its contribution to clinical decision-making needs to be evaluated in further studies.

\section{REFERENCES}

1 Greenberger PA. Allergic bronchopulmonary aspergillosis. J Allergy Clin Immunol 2002; 110: 685-692.

2 Kraemer R, Delosea N, Ballinari P, Gallati S, Crameri R. Effect of allergic bronchopulmonary aspergillosis on lung function in children with cystic fibrosis. Am J Respir Crit Care Med 2006; 174: 1211-1220.

3 Virnig C, Bush RK. Allergic bronchopulmonary aspergillosis: a US perspective. Curr Opin Pulm Med 2007; 13: 67-71.

4 Nelson LA, Callerame ML, Schwartz RH. Aspergillosis and atopy in cystic fibrosis. Am Rev Respir Dis 1979; 120: 863-873.

5 Stevens DA, Moss RB, Kurup VP, et al. Allergic bronchopulmonary aspergillosis in cystic fibrosis - state of the art: Cystic Fibrosis Foundation Consensus Conference. Clin Infect Dis 2003; 37: Suppl. 3, S225-S264.

6 Cunningham S, Madge SL, Dinwiddie R. Survey of criteria used to diagnose allergic bronchopulmonary aspergillosis in cystic fibrosis. Arch Dis Child 2001; 84: 89.

7 de Almeida MB, Bussamra MH, Rodrigues JC. Allergic bronchopulmonary aspergillosis in paediatric cystic fibrosis patients. Paediatr Respir Rev 2006; 7: 67-72.

8 Ratjen F, Doring G. Cystic fibrosis. Lancet 2003; 361: 681-689.

9 Burns JL, Emerson J, Stapp JR, et al. Microbiology of sputum from patients at cystic fibrosis centers in the United States. Clin Infect Dis 1998; 27: 158-163.

10 Skov M, McKay K, Koch C, Cooper PJ. Prevalence of allergic bronchopulmonary aspergillosis in cystic fibrosis in an area with a high frequency of atopy. Respir Med 2005; 99: 887-893.

11 Mastella G, Rainisio M, Harms HK, et al. Allergic bronchopulmonary aspergillosis in cystic fibrosis. A European epidemiological study. Eur Respir J 2000; 16: 464-471.

12 Hogaboam CM, Carpenter KJ, Schuh JM, Buckland KF. Aspergillus and asthma-any link? Med Mycol 2005; 43: Suppl. 1, S197-S202.
13 Hartl D, Latzin P, Zissel G, Krane M, Krauss-Etschmann S, Griese M. Chemokines indicate allergic bronchopulmonary aspergillosis in patients with cystic fibrosis. Am J Respir Crit Care Med 2006; 173: 1370-1376.

14 Casaulta C, Fluckiger S, Crameri R, Blaser K, Schoeni MH. Time course of antibody response to recombinant Aspergillus fumigatus antigens in cystic fibrosis with and without ABPA. Pediatr Allergy Immunol 2005; 16: 217-225.

15 Hemmann S, Nikolaizik WH, Schoni MH, Blaser K, Crameri R. Differential IgE recognition of recombinant Aspergillus fumigatus allergens by cystic fibrosis patients with allergic bronchopulmonary aspergillosis or Aspergillus allergy. Eur J Immunol 1998; 28: 1155-1160.

16 Salvatore F, Scudiero O, Castaldo G. Genotype-phenotype correlation in cystic fibrosis: the role of modifier genes. Am J Med Genet 2002; 111: 88-95.

17 Crameri R. Recombinant Aspergillus fumigatus allergens: from the nucleotide sequences to clinical applications. Int Arch Allergy Immunol 1998; 115: 99-114.

18 Nikolaizik WH, Moser M, Crameri R, et al. Identification of allergic bronchopulmonary aspergillosis in cystic fibrosis patients by recombinant Aspergillus fumigatus I/a-specific serology. Am J Respir Crit Care Med 1995; 152: 634-639.

19 Kurup VP, Banerjee B, Hemmann S, Greenberger PA, Blaser K, Crameri R. Selected recombinant Aspergillus fumigatus allergens bind specifically to IgE in ABPA. Clin Exp Allergy 2000; 30: 988-993.

20 Crameri R, Hemmann S, Ismail C, Menz G, Blaser K. Disease-specific recombinant allergens for the diagnosis of allergic bronchopulmonary aspergillosis. Int Immunol 1998; 10: 1211-1216.

21 Knutsen AP, Hutcheson PS, Slavin RG, Kurup VP. IgE antibody to Aspergillus fumigatus recombinant allergens in cystic fibrosis patients with allergic bronchopulmonary aspergillosis. Allergy 2004; 59: 198-203.

22 Kurup VP, Knutsen AP, Moss RB, Bansal NK. Specific antibodies to recombinant allergens of Aspergillus fumigatus in cystic fibrosis patients with ABPA. Clin Mol Allergy 2006; 4: 11.

23 Skov M, Pressler T, Jensen HE, Hoiby N, Koch C. Specific IgG subclass antibody pattern to Aspergillus fumigatus in patients with cystic fibrosis with allergic bronchopulmonary aspergillosis (ABPA). Thorax 1999; 54: 44-50.

24 Krane M, Griese M. Surfactant protein D in serum from patients with allergic bronchopulmonary aspergillosis. Eur Respir J 2003; 22: 592-595.

25 Shoseyov D, Brownlee KG, Conway SP, Kerem E. Aspergillus bronchitis in cystic fibrosis. Chest 2006; 130: 222-226.

26 Hartl D, Buckland KF, Hogaboam CM. Chemokines in allergic aspergillosis - from animal models to human lung diseases. Inflamm Allergy Drug Targets 2006; 5: 219-228.

27 Meier A, Kirschning CJ, Nikolaus T, Wagner $H$, Heesemann J, Ebel F. Toll-like receptor (TLR) 2 and TLR4 are essential for Aspergillus-induced activation of murine macrophages. Cell Microbiol 2003; 5: 561-570.

28 Vulcano M, Albanesi C, Stoppacciaro A, et al. Dendritic cells as a major source of macrophage-derived chemokine/ CCL22 in vitro and in vivo. Eur J Immunol 2001; 31: 812-822.

29 D'Ambrosio D, Iellem A, Bonecchi R, et al. Selective upregulation of chemokine receptors CCR4 and CCR8 upon 
activation of polarized human type 2 Th cells. J Immunol 1998; 161: 5111-5115.

30 Renauld JC. New insights into the role of cytokines in asthma. J Clin Pathol 2001; 54: 577-589.

31 Miyazaki E, Nureki S, Ono E, et al. Circulating thymusand activation-regulated chemokine/CCL17 is a useful biomarker for discriminating acute eosinophilic pneumonia from other causes of acute lung injury. Chest 2007; 131: 1726-1734.

32 Leung TF, Wong CK, Lam CWK, et al. Plasma TARC concentration may be a useful marker for asthmatic exacerbation in children. Eur Respir J 2003; 21: 616-620. 